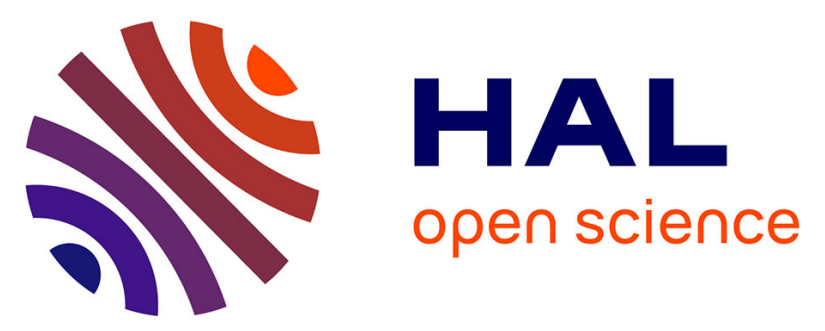

\title{
Control of the thermal hysteresis of the prototypal spin-transition FeII(phen)2(NCS)2 compound via the microcrystallites environment: experiments and mechanoelastic model
}

Antoine Tissot, Cristian Enachescu, Marie-Laure Boillot

\section{To cite this version:}

Antoine Tissot, Cristian Enachescu, Marie-Laure Boillot. Control of the thermal hysteresis of the prototypal spin-transition FeII(phen)2(NCS)2 compound via the microcrystallites environment: experiments and mechanoelastic model. Journal of Materials Chemistry, 2012, 22 (38), pp.20451. $10.1039 / \mathrm{c} 2 \mathrm{jm} 33865 \mathrm{c}$. hal-03325316

\author{
HAL Id: hal-03325316 \\ https://hal.science/hal-03325316
}

Submitted on 24 Aug 2021

HAL is a multi-disciplinary open access archive for the deposit and dissemination of scientific research documents, whether they are published or not. The documents may come from teaching and research institutions in France or abroad, or from public or private research centers.
L'archive ouverte pluridisciplinaire HAL, est destinée au dépôt et à la diffusion de documents scientifiques de niveau recherche, publiés ou non, émanant des établissements d'enseignement et de recherche français ou étrangers, des laboratoires publics ou privés. 


\title{
Control of the thermal hysteresis of the prototypal spin-transition
} $\mathrm{Fe}^{\mathrm{II}}$ (phen $)_{2}(\mathrm{NCS})_{2}$ compound via the microcrystallites environment: experiments and mechanoelastic model.

\author{
Antoine Tissot, ${ }^{a}$ Cristian Enachescu $^{b}$ and Marie-Laure Boillot ${ }^{* a}$
}

\begin{abstract}
Microcrystals of $\mathrm{Fe}^{\mathrm{II}}(\text { phen })_{2}(\mathrm{NCS})_{2}$ (phen $=1,10$-Phenanthroline), isolated by solvent-induced precipitation, were dispersed in glassy or semicrystalline matrices and subjected to thermal or chemical treatments. Interactions occurring between crystals and their macromolecular (or molecular) surroundings were revealed by drastic alteration of the first-order spin-transition afforded by this material. Depending on matrices and experimental treatments, the cooperativity in particles dispersion can be dampened, resulting in a more gradual transition or enhanced, providing a large hysteresis. The hysteresis broadening was also demonstrated with the incorporation of another first-order transition molecular material (bulk) in similar matrices. The different features associated to the in-polymers dispersions are accounted for in the framework of a mechanoelastic model based on a Monte-Carlo standard procedure.
\end{abstract}

\section{Introduction}

The dispersion of particles in a continuous phase (solid, liquid or gas) of different composition is a common process used in the elaboration of a wide range materials (suspensions, aerogels, emulsions, alloys, solutions...). The dispersion allows several characteristics (inter-particles interactions, concentration, reactivity...) to be finely controlled. It is also used in nanochemistry and therefore the description of structures and properties of these media is an active field of research. Our work, aiming at elaborating in a controlled and reproductible manner spin-transition nanoparticles (stabilization, separation of particles from each other or processing), has led us to investigate the physical properties of particles dispersed in polymeric phases, molecular oils or pastes.

The spin-crossover (SCO) compounds form a class of switchable molecule-based magnetic materials that exhibit remarkable properties of cooperativity and bistability in the solid state. ${ }^{1}$ The low-spin (LS) to high-spin (HS) transformation of $3 \mathrm{~d}^{4}-3 \mathrm{~d}^{7}$ transition metal complexes is achieved by means of various chemical or physical stimuli including temperature and pressure. In the HS species, the higher occupancy of antibonding orbitals (for pseudo-octahedral $\mathrm{Fe}^{\mathrm{II}}$ complexes: $\mathrm{LS}\left(\mathrm{t}_{2 \mathrm{~g}}\right)^{6}\left(\mathrm{e}_{\mathrm{g}}\right)^{0}$ and $\mathrm{HS}$ $\left.\left(\mathrm{t}_{2 \mathrm{~g}}\right)^{4}\left(\mathrm{e}_{\mathrm{g}}\right)^{2}\right)$ results in significant distorsion and expansion of molecules as well as of crystal volume. ${ }^{2}$ Long-range and shortrange cooperative interactions, mainly of elastic nature, derive from this molecular swelling leading to very abrupt transition and hysteretic effect. Due to the technological potentialities of such features, spin-transition molecular materials have attracted a peculiar attention and recent research work has been focused on size-related properties, processing and patterning. ${ }^{3}$

The methods applied for solids processing ${ }^{3,4}$ or miniaturization ${ }^{3,5}$ (water-in oil micro-emulsion...) require dispersion of compounds in suitable environments (polymer, surfactant...). Up to date, only few experimental ${ }^{6,7,8,9}$ and theoretical ${ }^{10,11}$ studies have addressed the question of the role played by the environment on the switching properties of SCO solids.

Here, we explore the mechanism by which the bistability of an iron(II) spin-transition compound may be affected by modifying the nature and the physical state of the dispersion medium. To avoid excessive damaging size-reduction effects, microparticles with small surface/volume ratio $\left(\leq 10^{-2}\right)$, were synthesized with a Fe(phen $)_{2}(\mathrm{NCS})_{2}$ compound (phen $=1,10$-Phenanthroline) exhibiting a first-order spin-transition (at ca. $176 \mathrm{~K} \dagger$ ). ${ }^{12,13 \mathrm{a}-\mathrm{b}}$ Advantage was taken of its non-ionic character for controlling the formation of particles with the sudden precipitation technique. As shown by some of us, ${ }^{14}$ this approach suits for the elaboration of spin-transition micro- and nanoparticles of purely molecular systems.

\section{Experimental}

\subsection{Syntheses}

[Fe(phen) $\left.{ }_{3}\right](\mathrm{NCS})_{2} .2 \mathrm{H}_{2} \mathrm{O}$ (noted 1) was synthesized as previously described. ${ }^{12}$ Elemental Analysis: Formula $\mathrm{C}_{38} \mathrm{H}_{28} \mathrm{~N}_{8} \mathrm{FeO}_{2} \mathrm{~S}_{2}$ (\%): Calcd: C 60.96, H 3.77, N 14,97; Found: C 61.66, H 3.88, N 14.65. IR (KBr): $v\left(\mathrm{NC}_{\mathrm{NCS}}\right)=2050 \mathrm{~cm}^{-1}$. The presence of two water molecules per metal ion was consistent with the $5-6 \%$ change of weight observed by TGA below $80{ }^{\circ} \mathrm{C}$. The red powder was characterized by X-ray diffraction pattern and IR spectrum (Fig. S1 and S2, ESI).

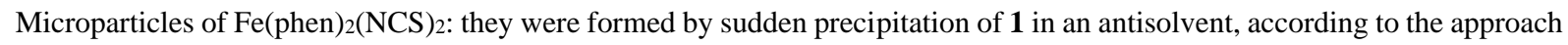
previously reported. ${ }^{14}$ The red solution obtained by dissolving $1(50 \mathrm{mg})$ in methanol $(4 \mathrm{~mL})$ was quickly added under vigorous stirring to butan-1-ol $(100 \mathrm{~mL})$ at $80{ }^{\circ} \mathrm{C}$. After $30 \mathrm{~min}$, a violet powder was isolated by centrifugation (10 min, $\left.10000 \mathrm{rpm}\right)$, washed with pentane and dried. The bare particles 2 were characterized by TEM. The images show a collection of thin rhombic 
platelets with an averaged size of $1700 \times 1700 \times 160 \mathrm{~nm}^{3}\left(\theta=76^{\circ}\right)$. Elemental analysis: Formula $\mathrm{C}_{26} \mathrm{H}_{16} \mathrm{FeN}_{6} \mathrm{~S}_{2}(\%)$ : Calcd: $\mathrm{C}$ 58.65, H 3.03, N 15.78, S 12.04; found: C 57.17, H 3.15, N, 15.21, S 12.17; IR(KBr): $v / \mathrm{cm}^{-1}: 2074,2062$ at $293 \mathrm{~K}$ and 2114 , 2106 at $99 \mathrm{~K}$ for $\mathrm{NC}_{\mathrm{NCSe}}$.

Microparticles of $\mathrm{Fe}(\text { phen })_{2}(\mathrm{NCS})_{2}$ encapsulated in matrices: Composite materials, noted 2-PEG or 2-PVP, were prepared by dispersing particles $2(10 \mathrm{mg}$ ) in a solution of butan-1-ol $(20 \mathrm{~mL}$ ) which contains $200 \mathrm{mg}$ of polymer (Polyethylene glycol, PEG, $M M=6000$ g.mol ${ }^{-1}$; Polyvinylpyrrolidone, PVP, $\left.M M=40000 \mathrm{~g} . \mathrm{mol}^{-1}\right)$. They were precipitated, either by cooling the solution down to $0^{\circ} \mathrm{C}$ (2-PEG) or by adding $40 \mathrm{~mL}$ of pentane (2-PVP); then they were centrifugated, washed and dried. Pastes were obtained by dispersing $1 \mathrm{mg}$ of $\mathbf{2}$ in $59.7 \mathrm{mg}$ of glycerol (nujol or eicosan after melting). The X-ray powder diffractograms of 2, 2-PEG, 2-PEG heated and 2-PVP were checked at $293 \mathrm{~K}$ (Fig. S1 in ESI). IR spectra of 1, 2 (at $R T$ and $100 \mathrm{~K}$ ) and 2PVP were also shown (Fig. S2).

\subsection{Physical measurements}

Powder X-ray diffraction diagrams were recorded at $293 \mathrm{~K}$ on a Philips Panalytical X'Pert Pro MPD powder diffractomer $(\mathrm{Cu}$ $\mathrm{K} \alpha_{1}, \lambda=1.5405 \AA$ ) equipped with a fast detector.

FT IR spectra were recorded on a Perkin-Elmer spectrometer (Spectrum 1000 ) equiped with the Eurolabo variable temperature cell (21525) and Specac temperature controller (20120).

Magnetic measurements were carried out using a Quantum Design SQUID magnetometer (MPMS5S Model) calibrated against a standard palladium sample. The magnetization $v s$. temperature data were collected at a $1 \mathrm{Kmin}^{-1}$ sweeping rate in the 5-300 $\mathrm{K}$ temperature range $(H=5000 \mathrm{Oe})$.

\section{Results and Discussion}

\subsection{Elaboration and characterization of microparticles}

In a first step, the cationic complex $\left[\mathrm{Fe}(\text { phen })_{3}\right](\mathrm{NCS})_{2} .2 \mathrm{H}_{2} \mathrm{O}$ (noted $\left.\mathbf{1}\right)$ was synthesized according to the reported procedure. ${ }^{12}$ The extraction of one phen ligand per formulae unit was carried out by quickly adding the red methanolic solution of $\mathbf{1}$, close to the supersaturation limit, to butan-1-ol at $80^{\circ} \mathrm{C}$ under vigourous stirring.

In Fig. 1, the TEM analysis of the violet powder, noted 2, shows the formation of rhombic platelets with 1700x $1700 \times 160 \mathrm{~nm}^{3}$ average sizes. The bare particles 2 were also dispersed in butan-1-ol in presence of polymer (PEG or PVP), then precipitated with pentane to yield an in-polymer dispersed compound (2-polymer). This treatment, as controlled by TEM, does not alter the average size and shape of objects.

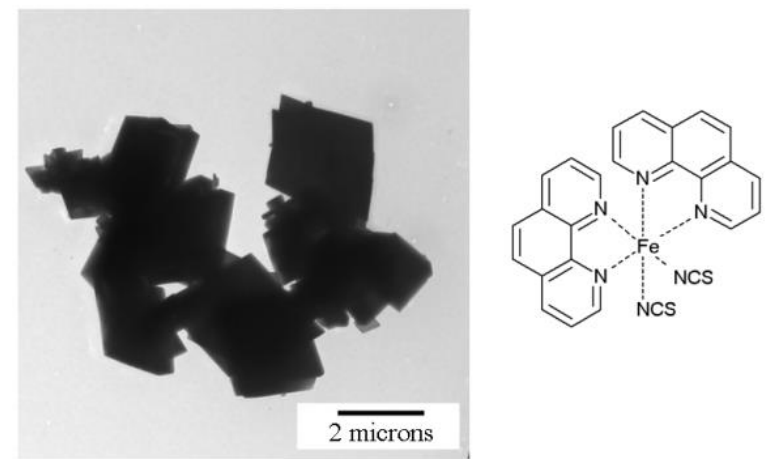

Fig. 1: (left) TEM image of powder 2; (right) molecular scheme of 2

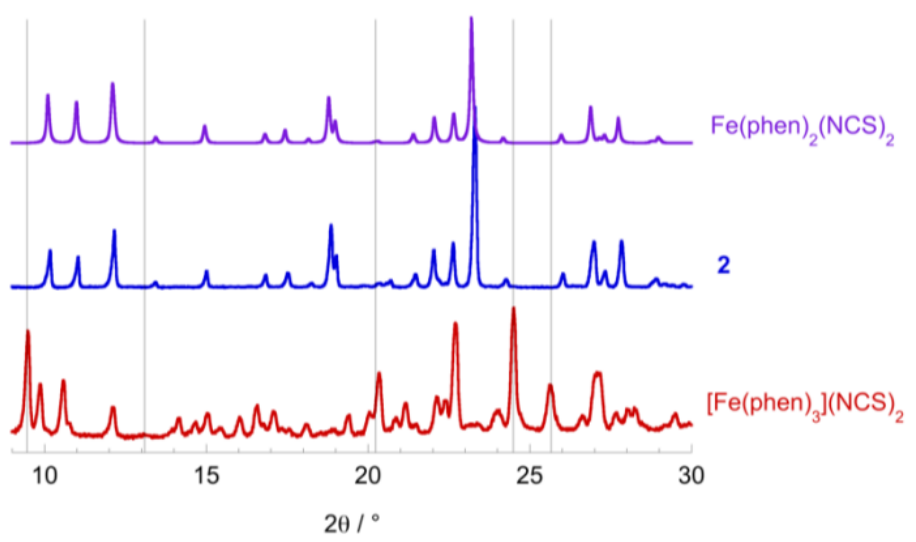

Fig. 2: X-ray powder diffractograms of 1 and 2 at $293 \mathrm{~K}$. The diffractogram of $\mathrm{Fe}(\mathrm{phen})_{2}(\mathrm{NCS})_{2}$ (polymorph II) in the HS phase calculated from lit. 13a is shown for comparison. 
The 293 K powder X-ray diffractogram of 2 (Fig. 2 and S1 in ESI) consists in a number of sharp reflections suggesting the formation of $\mathrm{Fe}(\text { phen })_{2}(\mathrm{NCS})_{2}$ in the form of HS microcrystals. ${ }^{13,15}$

The IR spectrum of $\mathbf{2}$ (Fig. S2) shows the absence of markers associated to $\mathbf{1}\left(\mathrm{v} / \mathrm{cm}^{-1}: 2050_{\mathrm{s}}, \mathrm{NC}_{\mathrm{NCSe}}\right)$ while new bands typical for $\mathrm{Fe}(\text { phen })_{2}(\mathrm{NCS})_{2}$ can be identified $\left(\mathrm{v} / \mathrm{cm}^{-1}: 2074 \mathrm{~s}, 2062 \mathrm{~s}\right.$ at $293 \mathrm{~K}$ and $2114_{\mathrm{s}}, 2106 \mathrm{~s}$ at $\left.99 \mathrm{~K}, \mathrm{NC}_{\mathrm{NCSe}}\right){ }^{16}$ This analysis, confirmed by elemental analysis, means that the insolubility of the cation $\mathbf{1}$ in butan-1-ol drives the extraction of one phen ligand from 1, i.e. a reaction commonly achieved by the soxhlet technique. In Fig. 3a, the temperature dependence of the $\chi_{\mathrm{M}} T$ product $\left(\chi_{\mathrm{M}}=\right.$ molar magnetic susceptibility, scan rate $=1 \mathrm{Kmin}^{-1}$ ) of 2 shows the almost complete and strongly cooperative $\mathrm{S}=0 \leftrightarrow \mathrm{S}=2$ transition of $\mathrm{Fe}$ (phen $)_{2}(\mathrm{NCS})_{2}\left(T_{1 / 2}=180 \mathrm{~K}\right.$, temperature at which the transition is half-completed; a very narrow hysteresis loop, if any, $\leq 0.4 \mathrm{~K} ; \chi_{\mathrm{M}} T=0.077(3.373) \mathrm{cm}^{3} \mathrm{~mol}^{-1} \mathrm{~K}$ at $\left.10(250) \mathrm{K}\right)$. On the whole, the magnetic behavior of microcrystals nicely compares with that of bulk (size of crystallites $>50 \mu \mathrm{m}$ ), and it may be concluded that reduction-size effects are negligible. The transition curve is not strictly identical to those reported for polymorphs I and II. $\dagger^{12,13}$

\subsection{Magnetic properties of composites}

In Fig 3a, the behaviors of 2-PEG or 2-PVP in the form of powders are compared to the bare particles one. The spin-transition characteristics ( $T_{1 / 2}$, cooperativity) of 2-PEG are almost unchanged with respect to 2 including a small HS residue ( $c a$. $\left.7 \%\right)$. In contrast, 2-PVP exhibits a broadening of the transition over the range between 250 and $140 \mathrm{~K}$ (i.e. starting at a higher temperature) and centered at $T_{1 / 2} \sim 173 \mathrm{~K}$. Since the composites are made up of strictly identical particles of micrometric size, interactions between particles and polymeric chains appear as the most relevant point to examine. Fig $3 \mathrm{~b}$ displays the $\chi_{\mathrm{M}} T v s$. $T$ curves for 2-PEG in different states: (i) the starting powder, (ii) the sample in-situ heated ( $350 \mathrm{~K}, 15 \mathrm{~min}$ ), (iii) the sample previously heated, then redispersed in butan-1-ol, reisolated by precipitation and dried. The melting and the cooling down of PEG $6000\left(T_{\mathrm{m}}=347 \mathrm{~K}\right)$ lead to a sample showing a progressive transition curve shifted toward smaller temperatures values $\left(T_{1 / 2} \sim 170 \mathrm{~K}\right)$. Interestingly, after the redispersion and reprecipitation steps of the heated composite, the magnetic behavior closely compares with the one of the non-heated material. This quasi-reversible evolution of 2-PEG precludes any alteration of the physical state (crystalline or punctual defects) of $\mathbf{2}$ that would be provoked by the thermal cycle and the related polymer behavior.

In Fig. 3c, the magnetic measurement was performed with microparticles 2 coated with glycerol, a glass-former liquid $\left(T_{\mathrm{m}}=\right.$ 291.2 K). The spin-transition of 2-glycerol occurs with a broad hysteresis loop; the ascending branch $(T \uparrow=180.5 \mathrm{~K})$ being steeper than the descending one $(T \downarrow=160.4 \mathrm{~K})$. The largest hysteresis $(20 \mathrm{~K}$ width) was produced by freezing the sample down to $10 \mathrm{~K}$. In the derivative curves, the transition peaks appear with additional shoulders on the high temperature side. Given that the glass-transition of glycerol is expected at $T_{\mathrm{g}} \sim 185-192 \mathrm{~K},{ }^{17}$ we suggest that the unresolved shoulders correspond to transitional states associated to the glycerol transformation (glass or supercooled liquid / viscoelastic phase). This suggestion is supported by the fact that the hysteresis loop slightly varies upon changing the scan rates $\left(0.5-1 \mathrm{Kmin}^{-1}\right)$ or cycling the temperature (Fig. S3). The variation of magnetization $v s$. time was recorded in the ascending and descending branches of the hysteresis loop (see Fig S4). At $180 \mathrm{~K}$, the very small changes observed within one hour (less than $2 \%$ ) are consistent with the bistable character of $\mathbf{2}$-glycerol in the hysteretic range. Compared to the microcrystals $\mathbf{2}$, the first-order transition of larger crystallites of $\mathrm{Fe}(\text { phen })_{2}(\mathrm{NCS})_{2}$ (size $>50 \mu \mathrm{m}$, powder of polymorph I) is also significantly impacted by the dispersion in glycerol (Fig S5, bulk $\mathrm{T} \downarrow \sim 177.9 \mathrm{~K}$ and $\mathrm{T} \uparrow \sim 178.2 \mathrm{~K}$, bulk-glycerol $\mathrm{T} \downarrow=169 \mathrm{~K}$ and $\mathrm{T} \uparrow=178 \mathrm{~K}$ ). This last finding indicates that the occurrence of the hysteresis effect is mainly determined by the bulk coupling between the spin-transition and the matrix properties.

The use of paraffinic oils of molecular nature -like eicosan or nujol $\left(\mathrm{C}_{\mathrm{n}} \mathrm{H}_{2 \mathrm{n}+2}\right.$, with $\mathrm{n}=20$ or large)- as coating matrices leads to new examples of asymmetrical hysteresis loops observed in the $\chi_{\mathrm{M}} \mathrm{T} v s$. T curves (Fig. 3d).

In a simple extension of this study, we have probed the behavior of another spin-transition material, a $\mathrm{Fe}^{\mathrm{III}}$ cationic complex $\mathrm{Fe}(3-\mathrm{MeO}-\mathrm{SalEen})_{2} \mathrm{PF}_{6}$ exhibiting a first-order transition, that some of us have investigated. ${ }^{14} \mathrm{In}$ Fig. S6, this ferric compound was studied in the form of large microcrystallites (bulk, size $>50 \mu \mathrm{m}$ ) and the dispersions of bulk in eicosan or glycerol. A qualitative agreement was found between the spin transition characteristics (low temperature shift, enhanced hysteresis effect) observed for $\mathrm{Fe}^{\mathrm{III}}(3-\mathrm{MeO}-\mathrm{SalEen})_{2} \mathrm{PF}_{6}$ (bulk $\mathrm{T} \downarrow \sim 162.3 \mathrm{~K}$ and $\mathrm{T} \uparrow \sim 163.4 \mathrm{~K}$, bulk-eicosan $\mathrm{T} \downarrow=155.5 \mathrm{~K}$ and $\mathrm{T} \uparrow=163.0 \mathrm{~K}$, bulkglycerol $\mathrm{T} \downarrow=153.5 \mathrm{~K}$ and $\mathrm{T} \uparrow=160.8 \mathrm{~K}$ ) and those above described for $\mathrm{Fe}(\mathrm{phen})_{2}(\mathrm{NCS})_{2}$.

The variety of magnetic behaviors -progressive or cooperative transition, bistability-, shows that both the surrounding medium of $\mathrm{Fe}(\text { phen })_{2}(\mathrm{NCS})_{2}$ crystals and experimental conditions (thermal cycle, scan rate, dispersion in a solvent) influence the spin transition. PVP, known as capping agent, as well as blends formed with glycerol or PEG, present adherence properties. Therefore the particles interface is subject to local effects that result from Van der Waals contacts with polymer segments or atom groups. ${ }^{18}$ Since Van der Waals forces take part in physisorption and capillarity phenomena, pressure effects can develop between the two components according to their local or macroscopic characteristics. To address this complex issue, a mecanoelastic approach was applied to the present observations. 


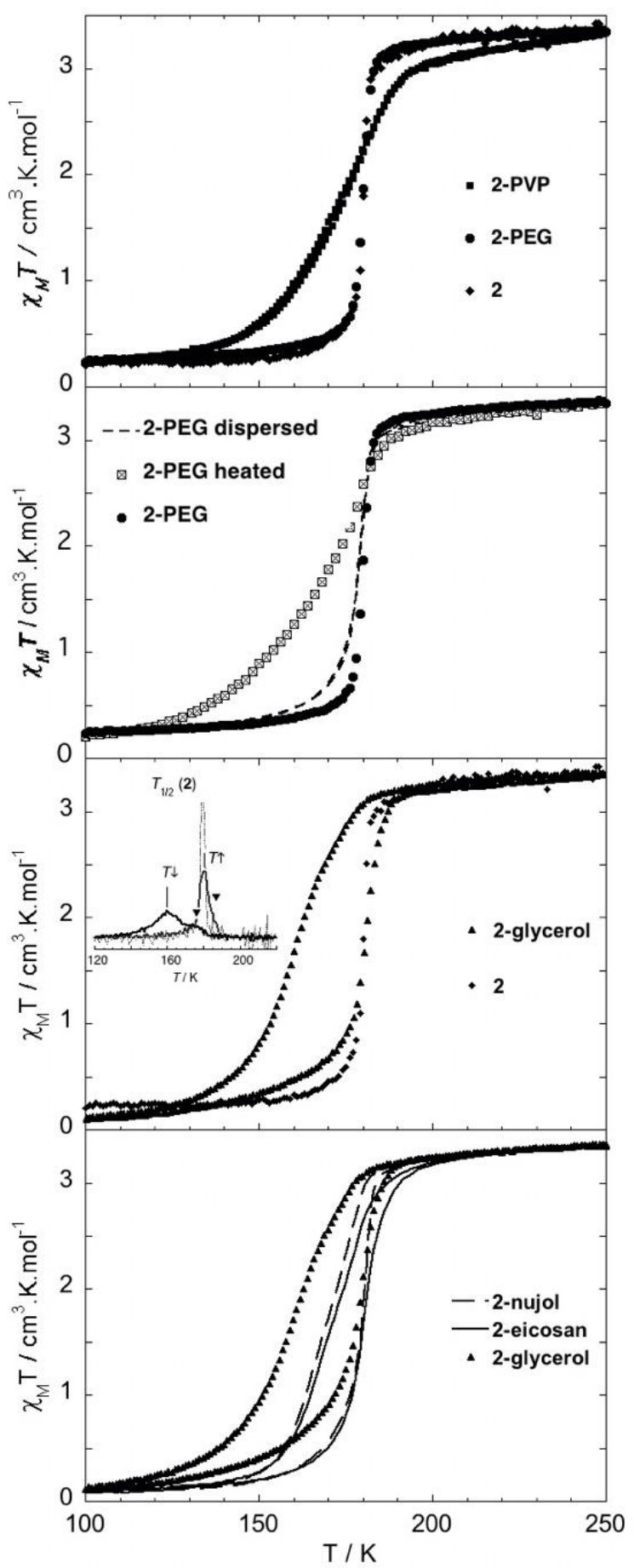

Fig. 3: Magnetic properties of microcrystals measured at a scan rate of $1 \mathrm{Kmin}^{-1}$ : (a) bare particles 2, 2-PEG and 2-PVP composites formed by precipitation in BuOH; (b) 2-PEG before and after heating, 2-PEG heated, then redispersed in $\mathrm{BuOH}$ and precipitated ; (c) 2 and particles dispersed in glycerol, 2-glycerol. The sample was first freezed at $10 \mathrm{~K}$ and then studied from $70 \mathrm{~K}$ at $1 \mathrm{Kmin}^{-1}$. In the derivatives curves (inset), the marks indicate transition peaks of $\mathbf{2}$ and 2-glycerol; (d) particles dispersed in nujol 2-nujol, eicosan after melting 2-eicosan and glycerol 2-glycerol.

\subsection{The mechanoelastic analysis}

Even though experimental data concerning the effect of environment ${ }^{9 \mathrm{a}}$ and particle size on the thermal spin transition have been published, ${ }^{5,8,9}$ very few theoretical attempts have been made in order to rationalize these effects. Ising-like models for open boundary systems (i) with forced HS molecules at the edge ${ }^{19}$ or (ii) embedded in polymers (so interacting with edge spincrossover molecules),${ }^{20}$ had reproduced some of the experimental features. However they were not directly associated with experiments and their use for small spin-crossover systems were limited, as they did not take into account the distortions that originate from the different molecular size in the two HS and LS states. Very recently, a first attempt to understand the thermal transition in the case of spin-crossover nanoparticles in various environments has been made in the framework of the 
mechanoelastic model, ${ }^{11}$ but, as in the Ising-like models case, it has not been directly applied to genuine experimental data. The recently developed mechanoelastic (ME) model $^{10}$ is based on the so-called ball and spring concept and implies that the elastic interactions inside the sample arise from lattice distortion due to the difference of the molecular sizes between the LS and HS states. The SCO molecules are represented as rigid spheres, situated in open boundary lattices and interacting via connecting springs. The variation of volume of a switching molecule produces an instantaneous elastic force on the neighboring springs and consequently determines a shift in initial position of the closest neighbors, hence of all other molecules in the system. Therefore, the volume and shape of the whole system change with every molecular switch. The non-periodical conditions intrinsic to the ME model make it appropriate for the study of SCO samples embedded in various environments. As mentioned above, the preliminary theoretical study on the thermal hysteresis of SCO nanoparticles embedded in polymer ${ }^{11}$ has been able to reproduce the main experimental features encountered in our work: a smoother thermal transition, sometimes incomplete, shifted towards lower temperatures and with a narrower hysteresis, if any.

In order to study the thermal transition within the ME model, one assigns for every SCO molecule a HS $\rightarrow$ LS or a LS $\rightarrow$ HS transition probability, depending on the temperature $T$, on intrinsic material parameters (the HS-LS energy difference $D$, the degeneracy ratio $g$, the effective activation energy $E_{\boldsymbol{A}}$ ) and on the interactions between molecules, represented here by the way of the local pressure $p_{i}$, defined as the sum of elastic forces acting on the molecule according to:

$$
\begin{gathered}
P_{H S \rightarrow L S}^{i}=\frac{1}{\tau} \exp \left(-\frac{E_{A}-\kappa p_{i}}{k_{B} T}\right) \\
P_{L S \rightarrow H S}^{i}=\frac{1}{\tau} \exp \left(-\frac{D-k_{B} T \ln g}{k_{B} T}\right) \exp \left(-\frac{E_{A}-\kappa p_{i}}{k_{B} T}\right)
\end{gathered}
$$

where $\tau$ a constant scaling factor, and $\kappa$ a constant that establishes to which extent the local pressure influences the relaxation. Following a Monte-Carlo standard procedure, one decides if a molecule switches or no. A Monte-Carlo step (MCS) is completed when every molecule has been checked once. After every MCS, all molecules are allowed to move in order to find their new equilibrium positions, implying other local interactions.

For the simulations presented below, we have used a $10^{5} \mathrm{MCS}$ at every temperature and a hexagonal lattice composed of 2791 molecules linked by identical springs with the elastic constant $k=1 \mathrm{~N} / \mathrm{m}$. These values lead to simulated curves close to the quasistatic regime for the bulk system, as discussed in lit. ${ }^{10}$.

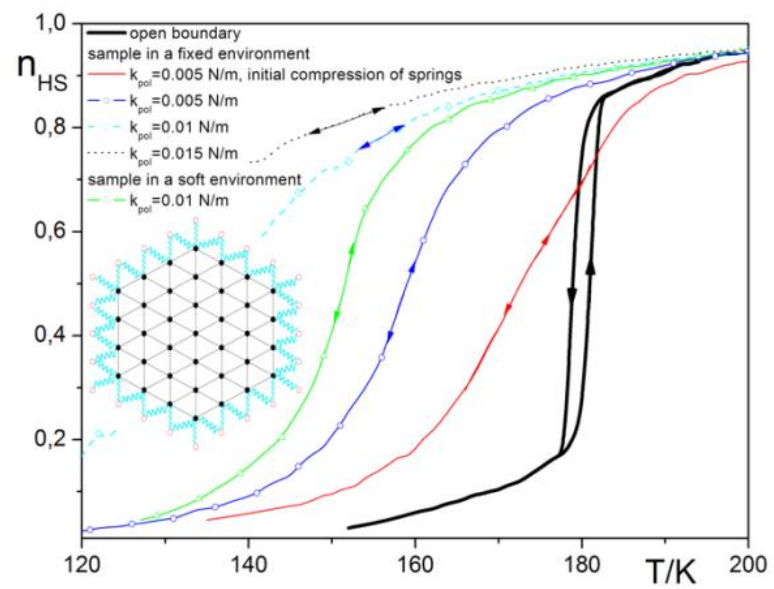

Fig. 4: Comparison between thermal transition for an open boundary system and for systems embedded in a rigid or partially soft environment. In the inset: the hexagonal lattice composed of SCO molecules (black circles) interconnected by springs (lines) embedded in polymer represented as neutral molecules (open circles), connected to SCO molecules at the edge by springs (zigzag lines). The intrinsic system parameters are marked in text; the other parameters are: $k=1 \mathrm{~N} / \mathrm{m}, E_{A}=400 \mathrm{~K}, \kappa=1800$.

Considering open boundary conditions, and intrinsic parameters corresponding to experimental data available for bulk $\mathrm{Fe}(\text { phen })_{2}(\mathrm{NCS})_{2}{ }^{12 \mathrm{~b}}(\Delta H=1045 \mathrm{~K}, g=330)$ a small hysteresis loop similar to the experimental one is obtained (Fig. 4). In order to reproduce the other experimental data, we consider weak interactions between the edge SCO molecules and the surrounding medium. For practical reasons, this boundary effect is simulated by adding in the vicinity of the hexagonal lattice a shell of molecules, which interact with the $\mathrm{Fe}^{\mathrm{II}}$ complexes situated at the edge of the lattice by the way of springs, with a small elastic constant, $k_{\text {pol }}$ (inset of Fig.4). If the polymer chain is rigid, the reduction of the overall volume of the lattice induces an increasing negative pressure at the edge of the system that finds its origin in the backpulling forces of the polymer network. This will progressively reduce the HS-LS switching probability of the SCO molecules and will result basically in a less steep transition curve with a lower $T_{1 / 2}$ depending on $k_{p o l}$ (Fig. 4). In order to obtain a transition curve, which starts at a higher temperature, as in the PVP experimental case, we consider an initial compression of the springs linking edge molecules with 
the polymer, thus creating an initial positive external pressure. As the transition proceeds, this pressure decreases, passes through zero and then becomes increasingly negative, leading to a gradual transition curve, with a higher $T_{1 / 2}$ than previously. If the polymeric shell is completely soft, and no initial pressure is applied to the lattice, then a thermal transition identical to open boundary case is expected. A situation, in which the polymer is partially soft, can also be considered. For the same value of $k_{\mathrm{pol}}$, it leads to an intermediate transition curve in-between rigid polymer and soft polymer cases.

In view of these results, some experimental observations can be shortly commented and further issues can be drawn.

\subsection{Discussion}

The spin-transition phenomenon is known to proceed through electron-phonon coupling ${ }^{21}$ and to be impacted by cooperative interactions of elastic nature. ${ }^{22} \mathrm{In} \mathrm{Fe}(\mathrm{phen})_{2}(\mathrm{NCS})_{2},{ }^{13}$ the latter are mediated by a 3D network of strong intermolecular contacts giving rise to a steep transition and a weak thermal hysteresis. The dilution of such a cooperative solid in an inert crystalline matrix produces a progressive conversion rationalized in the frame of the mean-field approach by a variable internal pressure. Positive (negative) pressure values shift the transition curve towards higher (lower) temperature values as a consequence of the relative stabilization of the LS (HS) state. ${ }^{13,23}$

In the present work, the effects experienced by $\mathrm{Fe}(\text { phen })_{2}(\mathrm{NCS})_{2}$ microcrystals in the dispersion media can be analyzed as associated to variable but essentially negative pressures. However, the observance of a bistable regime of $\mathbf{2}$ in presence of glycerol (or eicosan, nujol) is a striking result that leads us to distinguish the particles coating: macromolecular vs. molecular matrices.

The transition curve of 2-PVP qualitatively agrees with the literature as a decrease in both temperature and steepness (cooperativity) is reported for spin-transition compounds ${ }^{4}$ or nanoparticles ${ }^{7,8,9}$ dispersed in polymers (or surfactants). Our data in Fig 3a-b also reveal the importance of polymer nature and sample history (treatment or elaboration). The behaviors of $\mathbf{2 , 2}$, PEG and 2-PEG redispersed are closely similar what suggests a weak affinity of macromolecules for the bare microcrystals and a small number of contacts. This property, previously noted in our study of in-PEG nano- and microparticles of a $\mathrm{Fe}^{\mathrm{III}}$ molecular salt,,${ }^{14}$ is no longer true when the polymer is melted and solidified around particles i.e. for 2-PEG heated. The fusion of PEG makes possible the adsorption at the crystal surface of multiple segments of polymeric chains, ${ }^{18}$ a feature leading to a denser composite, a larger surface saturation and the reinforcement of interfacial effects. The SCO curve of 2-PEG heated compares the one of 2-PVP, in which the adhesive properties of PVP also favor multiple anchoring of macromolecules at surface. The mechanical characteristics of PEG are associated to the formation of semi-crystalline phases below $\mathrm{T}_{\mathrm{m}} \sim 347 \mathrm{~K} .^{24}$ The larger the crystalline areas, as here characterized for 2-PEG heated, the stiffer the coating polymer. PVP in a glassy state below $\mathrm{T}_{\mathrm{g}} \sim 458 \mathrm{~K},{ }^{25}$ is rigid and not easily deformable. Upon the HS-to-LS conversion, the shrinkage of particles volume $(\sim 3$ $\%)^{13}$ carry the segments of adsorbed polymers. For chains weakly connected to the surface and locked by the matrix (2-PEG and 2-PEG redispersed), we can expect a spin-transition behavior close to the one of the uncoated particles $\mathbf{2}$. For an efficient anchoring of chains, antagonist forces (attractive Van der Waals forces similar to deformable springs) at surface have to be considered (negative pressure), as well as steric interchains ${ }^{18}$ effect (positive pressure). The mecanoelastic model satisfyingly reproduces the properties of 2-PVP and 2-PEG heated considering a rigid shell of polymers with an initial positive pressure giving rise to a progressive transition shifted towards lower temperature values.

Replacing PEG by glycerol molecules gives rise to a bistable behavior of 2-glycerol. Glycerol bears OH functions forming intra- and intermolecular hydrogen bonds. At $\sim 291 \mathrm{~K}$, the liquid solidifies and becomes a visco-elastic matrix, whereas below 185-192 K, it turns to a glass with specific and somewhat complex properties (viscosity, density, relaxation dynamics, fragility...). ${ }^{17}$ The fact that on the cooling cycle the spin transition of $\mathbf{2}$-glycerol occurs at a lower temperature than $\mathbf{2}$, again agrees with particles suffering negative pressures and thus can be modeled by a rigid matrix (fixed environment) interacting with particles. In the heating cycle, analysis with the same set of parameters fails to reproduce the ascending branch of hysteresis. Therefore we may suggest as hypotheses that the composite material has suffered an alteration in the low temperature range: a variation of volume due to the matrix itself or microscopic changes at the interfaces. The second point comes from the similarity between the ascending branches of transitions of 2-glycerol and 2 (also observed with 2-nujol or 2-eicosan and 2). It may be attributed to a quantitative disappearance of glycerol-particles interactions. As the glycerol environment is rigid $\left(\mathrm{T}<\mathrm{T}_{\mathrm{g}}\right.$ ), a thermal contraction of microcrystals volume of $\sim 3 \%$ (upon the HS to LS conversion) is large enough to produce a lengthening of glycerol-particles distances $\geq 5 \AA$ and thus, the breaking of Van der Waals contacts at interfaces. The LS lattice contraction (extrapolated at $\sim 3 \%$ between 180 and $10 \mathrm{~K})^{13}$ should also establish this low-temperature effect. The observation of an almost reproducible hysteresis loop over successive thermal cycles indicates that the heating of the sample above $180 \mathrm{~K}$, i.e. temperature corresponding to a fully HS particles volume, restores the glycerol environment at interfaces. At this point, other features to consider here are the remarkable changes (suppressed mobility of molecules, huge enhancement of stiffness, increase of density) of the glass-forming liquid occuring below $T_{\mathrm{g}}$, i.e. just in the range of the thermal SCO. The glass transition is commonly accompanied by an hysteresis effect because the structural relaxation (restoration of configurational motions) takes place on heating slightly below $T_{\mathrm{g}} .{ }^{26}$ From the data collected on heating, the rather cooperative spin-transition slightly precedes or is coupled to this relaxation and glass transition. Clearly, both the use of a molecular liquid and the proximity between the spin transition and the glass transition of glycerol are two key features of this result.

To the best of our knowledge, no glass transition is established for pure eicosan or nujol matrices, which likely evolve with formation of crystalline heterogeneities. The magnetic behavior of related composites is similar to the glycerol one with aperture of a smaller hysteresis loops (Fig 3d), a feature that indicates that the glass transition of the matrix is not a pre-requisite 
for the observation of a thermal bistability. It emphasizes the complexity of the spin-transition behaviors when spin-transition crystallites are dispersed in molecular matrices.

\section{Conclusions}

Using the well known Fe(phen) $)_{2}(\mathrm{NCS})_{2}$ compound, whose first-order spin transition is mainly governed by elastic interactions, the present work brings out the importance of supramolecular forces between particles interfaces and neutral coating agents. Following their encapsulation, microcrystals of $\mathrm{Fe}(\mathrm{phen})_{2}(\mathrm{NCS})_{2}$ exhibit a thermal bistability (2-glycerol or 2-eicosan, 2nujol), an essentially unaffected cooperative HS $\leftrightarrow$ LS (2-PEG) or a progressive conversion (2-PVP or 2-PEG heated). A reduction of transition temperatures with respect to bare particles and a spreading of processes on the low- and also hightemperature range may also be observed. These remarkable behaviors can be rationalized by considering mechanoelastic effects associated to particles environments. The results show their modulation by the matrix nature (molecular length, ability to establish contacts, ...), thermal and chemical treatments (melting and cooling of molecules) and some physical properties (stiffness, glass transition,...) in the transition temperature range.

The present investigation, dealing with the issue of nanoscale interfacial interactions in condensed matter, a very active field of biophysics, ${ }^{27}$ photophysics, ${ }^{28}$ nanotechnology ${ }^{18}$ or soft-matter science, ${ }^{18,29}$ disclose a valuable insight into the multiscale character of the spin-transition phenomenon.

The observation of a variable bistability of $\mathrm{Fe}(\mathrm{phen})_{2}(\mathrm{NCS})_{2}$ or $\mathrm{Fe}(3-\mathrm{MeO}-\mathrm{SalEen})_{2} \mathrm{PF}_{6}$, two prototypal spin-transition compounds with distinct characteristics (neutral $\mathrm{Fe}^{\mathrm{II}}$ or cationic $\mathrm{Fe}^{\mathrm{III}}$ species), in presence of a semi-crystalline / glass-forming molecular matrix opens a route for enhancing via the environment, the hysteretic behavior and thus, optimizing the bistable materials processed at micro-, nanoscales ${ }^{5,8,9,14,30}$ or even, nanopatterned. ${ }^{3,31}$

Advantages have been taken here on the molecular nature of the spin-transition material and the micrometric size of particles. For non-molecular SCO materials, i.e. coordination 3D networks (covalently interconnected spin-transition sites), we can suggest that the saturation of coordination sites at nanoparticles surfaces and electrostatic forces (residual salts, surfactants, ...) might result in additional contributions.

\section{Acknowledgements}

This work was supported by the CNRS, the French Ministery of research, ANR (ANR-09-BLAN-0212). CE thanks the Romanian CNCS, projects PCCE 9/2010 and RU-TE 185/2010. AT and MLB warmly thank Sandra Mazerat, Dr. Eric Rivière and Pr. René Clément for their kind assistance. They also thank Thibaud Maillard for his contribution in the syntheses.

\section{Notes and references}

${ }^{a}$ ICMMO, ECI, UMR CNRS 8182, Université Paris-Sud 11, 91405 Orsay cedex, France. Fax: 33 16915 4754; Tel: 33 16915 4755; E-mail: marie-laure.boillot@u-psud.fr.

${ }^{b}$ Faculty of Physics, "Alexandru Ioan Cuza" University, 7005506, Iasi, Romania.

$\dagger$ Electronic Supplementary Information (ESI) available: [Syntheses and characterizations, (S1) X-ray powder diffractograms, (S2) IR spectra, (S3-5) Magnetic behaviors of the different samples of Fe(phen $)_{2}(\mathrm{NCS})_{2}$, (S6) Magnetic behavior of $\left.\mathrm{Fe}(3-\mathrm{MeO}-\mathrm{SalEen})_{2} \mathrm{PF}_{6}\right]$.

See DOI: $10.1039 / \mathrm{b} 000000 \mathrm{x} /$

$\$ \dagger$ Depending on synthetic conditions, two polymorphs of $\mathrm{Fe}(\mathrm{phen})_{2}(\mathrm{NCS})_{2}$ were reported. Their spin-transition processes were characterized by specific magnetic, thermodynamical and structural features. Polymorph I obtained by extraction shows a complete first-order phase transition $(\mathrm{T} \downarrow=176.15$ and $\mathrm{T} \uparrow=176.30 \mathrm{~K})$. Polymorph II exhibits a process centered at $(\mathrm{T} \downarrow=178$. and $\mathrm{T} \uparrow=179.5 \mathrm{~K})$ with a slightly weaker cooperative character and some HS residue. (see ref 13)

(a) O. Kahn in Molecular Magnetism, Wiley-VCH, New-York, 1993; (b) Spin-crossover in transition metal compounds, I-III, in Topics in Current Chemistry, ed. P. Gütlich and H. A. Goodwin, Springer, Berlin, 2004, vol. 233-235.

(a) C. Chong, A. Slimani, F. Varret, K. Boukheddaden, E. Collet, J.-C. Ameline, R. Bronisz, A. Hauser, Chem. Phys. Lett., 2011, 504, 13, 29-33; (b) F. Varret, F. Slimani, K. Boukheddaden, C. Chong, H. Mishra, E. Collet, J. G. Haasnoot, S. Pillet, New J. Chem., 2011, 35, $10,2333$.

A. Bousseksou, G. Molnar, L. Salmon and W. Nicolazzi, Chem. Soc. Rev., 2011, 40, 3313.

A. Hauser, J. Adler and P. Gütlich, Chem. Phys. Lett., 1988, 152, 468.

(a) E. Coronado, J. R. Galan-Mascaros, M. Monrabal-Capilla, J. Garcia-Martinez and P. Pardo-Ibanez, Adv. Mater., 2007, 19, 1359; (b) T. Forestier, S. Mornet, N. Daro, T. Nishihara, S. Mouri, K. Tanaka, O. Fouche, E. Freysz and J.-F. Létard, Chem. Commun., 2008, 4327; (c) T. Forestier, A. Kaiba, S. Pechev, D. Denux, P. Guionneau, C. Etrillard, N. Daro, E. Freysz and J.-F. Létard, Chem.-Eur. J., 2009, 15, 6122; (d) J. R. Galan-Mascaros, E. Coronado, A. Forment-Aliaga, M. Monrabal-Capilla, E. Pinilla-Cienfuegos and M. Ceolin, Inorg. Chem., 2010, 49, 5706; (e) L. Salmon, G. Molnar, D. Zitouni, C. Quintero, C. Bergaud, J.-C. Micheau and A. Bousseksou, J. Mater. Chem., 2010, 20, 5499; (f) I. Boldog, A. B. Gaspar, V. Martinez, P. Pardo-Ibanez, V. Ksenofontov, A. Bhattacharjee, P. Gütlich and J. A. Real, Angew. Chem. Int. Ed., 2008, 47, 6433; (g) C. Bartual-Murgui, N. A. Ortega-Villar, H. J. Shepherd, M. C. Munoz, L. Salmon, G. Molnar, A. Bousseksou and J.-A. Real, J. Mater. Chem., 2011, 21, 7217; (h) J. Larionova, L. Salmon, Y. Guari, A. Tokarev, K. Molvinger, G. Molnar and A. Bousseksou, Angew. Chem. Int. Ed., 2008, 47, 8236.

6 M.-L. Boillot, S. Pillet, A. Tissot, E. Rivière, N. Claiser and C. Lecomte, Inorg. Chem., 2009, 47, 4729.

(a) A. Tokarev, L. Salmon, Y. Guarri, W. Nicolazzi, G. Molnar and A. Bousseksou, Chem. Commun., 2010, 46, 8011; (b) A. Tokarev, L. Salmon, Y. Guarri, G. Molnar and A. Bousseksou, New J. Chem., 2011, 35, 2081.

8 V. Martinez, I. Boldog, A. B. Gaspar, V. Ksenofontov, A. Bhattacharjee, P. Gütlich and J. A. Real, Chem. Mater., $2010,22,4271$. 
9 (a) Y. Raza, F. Volatron, S. Moldovan, O. Ersen, V. Huc, C. Martini, F. Brisset, A. Gloter, O. Stefan, A. Bousseksou, L. Catala and T. Mallah, Chem. Commun., 2011, 47, 11501; (b) F. Volatron, L. Catala, E. Rivière, A. Gloter, O. Stéphan and T. Mallah, Inorg. Chem., 2008, 47, 6584 .

10 (a) C. Enachescu, L. Stoleriu, A. Stancu and A. Hauser, Phys. Rev. Lett. 2009, 102, 257204; (b) C. Enachescu, M. Nishino, S. Miyashita, L. Stoleriu, A. Stancu and A. Hauser, Europhys. Lett., 2010, 91, 27003.

11 L. Stoleriu, P. Chakraborty, A. Hauser, A. Stancu and C. Enachescu, Phys. Rev. B, 2011,84 (13), 134102.

12 (a) E. König and K. Madeja, Inorg. Chem., 1967, 6, 48; (b) M. Sorai, J. Phys. Chem. Solids, 1974, 35, 555; (c) P. Ganguli, P. Gütlich and E. W. Müller, J. Chem. Soc. Dalton Trans., 1981, 441.

13 (a) B. Gallois, J.-A. Real, C. Hauw and J. Zarembowitch, Inorg. Chem., 1990, 29, 1152; (b) J.-A. Real, B. Gallois, T. Granier, F. SuezPanama and J. Zarembowitch, Inorg. Chem., 1992, 31, 4972; (c) C. Roux, J. Zarembowitch, J.-P. Itié, A. Polian and M. Verdaguer, Inorg. Chem., 1996, 35, 574.

14 A. Tissot, L. Rechignat, A. Bousseksou and M.-L. Boillot, J. Mater Chem., 2012, 22, 3411.

15 G. Lebedev, S. Pillet, C. Baldé, P. Guionneau, C. Desplanches and J.-F. Létard, IOP Conf. Ser.: Mater. Sci. Eng., $2009,5,012025$.

G. Brehm, M. Reiher and S. Schneider, J. Phys. Chem. A, 2002, 106, 12024.

7 R. Chelli, P. Procacci, G. Cardini, R. G. Della Valle and S. Califano, Phys. Chem. Chem. Phys., 1999, 1, 871.

1 B. A. Rozenberg and R. Tenne, Prog. Polym. Sci., 2008, 33, 40. J. Nasser, Eur. Phys. J. B, 2001, 21, 3. H. Spiering, Top. Curr. Chem., 2004, 235, 171.

19 (a) A. Muraoka, K. Boukheddaden, J Linarès and F. Varret, Phys Rev B, 2011, 84, 054119

20 A. Atitoaie, R. Tanasa and C. Enachescu, J. Mag. Mag. Mat., 2012, 324, 1596.

1 J. Nasser, Eur. Phys. J. B, 2001, 21, 3.

22 H. Spiering, Top. Curr. Chem., 2004, 235, 171.

23 (a) J.-P. Martin, J. Zarembowitch, A. Bousseksou, A. Dworkin, J.G. Haasnoot and F. Varret, Inorg. Chem., 1994, 33, 6325; (b) A. Hauser, N. Amstutz, S. Delahaye, A. Sadki, S. Schenker, R. Sieber and M. Zerara, in Optical spectra and chemical bonding in inorganic compounds, Vol 1, Struct. Bond., 2004, 106, pp. 81.

24 J. M. Ginés, M.J. Arias, A. M. Rabasco, C. Novák, A. Ruiz-Conde and P. J. Sánchez-Soto, J. Therm. Anal., 1996, 46, 291.

5 S. Fitzpatrick, J. F. McCabe, C. R. Petts and S. W. Booth, Int. J. Pharm., 2002, 246, 143.

J. C. Dyre, Rev. Mod. Phys., 2006, 78, 953.

27 T. E. Dirama and G. A. Carri, in Multiscale Simulation Methods for Nanomaterials, ed R. B. Ross and S. Mohanty, J Wiley \& Sons, Hoboken, NJ, USA, 2008, ch. 6, pp 89-125.

28 F. Kulzer, T. Xia and M. Orrit, Angew. Chem. Int. Ed., 2010, 49, 854.

D. H. Napper in Colloid Science, Academic Press, New-York, 1983.

R. Bertoni, M. Lorenc, A. Tissot, M. Servol, M.-L. Boillot and E. Collet, Angew. Chem., Int. Ed. (doi: 10.1002/anie.201202215)

31 M. Cavallini, I. Bergenti, S. Milita, G. Ruani, I. Salitros, Z-R. Qu, R. Chandrasekar and M. Ruben, Angew. Chem., Int. Ed., 2008, 47, 8596. 